\title{
Inguinoscrotal hernias involving urologic organs: A case series
}

\author{
Jeffrey Peter McKay, MD; ${ }^{*}$ Michael Organ, MD; ${ }^{*}$ Scott Bagnell, MD; ${ }^{*}$ Christopher Gallant, MD; ${ }^{\dagger}$ \\ Christopher French, MD, FRCSC ${ }^{\S}$
}

*Department of Urology, Dalhousie University, Halifax, NS; 'Department of Family Medicine, Dalhousie University, Halifax, NS; §Department of Urology, Memorial University, St. John's, NL

Cite as: Can Urol Assoc J 2014;8(5-6):e429-32. http://dx.doi.org/10.5489/cuaj.225 Published online June 19, 2014.

\section{Abstract}

We report 2 cases of inguinoscrotal hernias involving urologic organs. The first case involved an elderly gentleman with a history of micturition by squeezing his scrotum. He was diagnosed as having a right-sided indirect inguinal hernia involving the right ureter and bladder. Treatment was surgical. The second case involved an achondroplastic male who presented with acute kidney injury. He had bilateral hydronephrosis and ureteric obstruction secondary to an ureteroinguinal herniation bilaterally. The presentation, diagnosis, and treatment of inguinoscrotal hernias involving the bladder and ureters are discussed.

\section{Introduction}

As of 2003, there have been 190 reported cases of inguinal hernias containing urologic organs. ${ }^{1}$ Massive bladder herniation into the scrotum is rare. Presentation is often with double phase micturition, urgency, frequency, nocturia and hematuria. $^{2}$ The preferred treatment is surgery. We present a case of a 78-year-old male who described difficulties with micturition for 10 years, but other symptoms led to his diagnosis of inguinoscrotal bladder herniation. Ureteroinguinal herniation is also very rare, with 140 reported cases as of April 2009. ${ }^{3}$ The second case involves an achondroplastic male with bilateral ureteroinguinal herniations causing obstruction. We discuss the presentation, imaging studies and treatment of these conditions.

\section{Case 1}

A 78-year-old male with a swollen scrotum presented to the emergency room with a 2-day history of increasing right groin pain. Pain was positional and noted to be worse when sitting and standing and relieved when supine. Associated features included nausea and difficulty initiating micturition. The patient elevated and squeezed his scrotum to initiate voids. Medical history included hypertension, arthritis, and psoriasis. He had no previous surgeries and his body mass index (BMI) was normal. On examination, his abdomen had an umbilical hernia, but was otherwise unremarkable. Examination of his scrotum revealed a grapefruit-sized, right hemiscrotal mass originating above the inguinal ligament. The mass was positive for transillumination and was nontender to palpation. There was no positional change in size. Laboratory investigations were unremarkable. Given the presentation, history and physical exam, the decision was made to obtain a computerized tomography $(\mathrm{CT})$ scan of the abdomen and pelvis with contrast. The CT demonstrated a large, right-sided hernia of the right ureter and bladder into the inguinal canal and scrotum (Fig. 1).

After 2 days in hospital, the patient's pain resolved. He was discharged and returned to have surgery (Fig. 2).

Under general anesthesia, the patient underwent a laparotomy with scrotal access. The bladder was removed from the scrotum. A portion of the bladder appeared ischemic requiring partial cystectomy and bladder repair. The ureter appeared long and redundant. Due to the associated risk of ureter folding and kinking, the distal end was excised and the ureter reimplanted. Filling the bladder intraoperatively demonstrated no leakage. An inguinal hernia was subsequently repaired using Prolene mesh (Ethicon Endo-Surgery, Inc.) by General Surgery.

The patient was able to void successfully after the operation. Uroflowmetry and cystoscopy were consistent with mild obstruction from the prostate. In the early postoperative phase, the patient had right-sided vesicoureteral reflux and suffered from multiple urinary tract infections. He was placed on prophylactic nitrofurantoin without any further complications. 


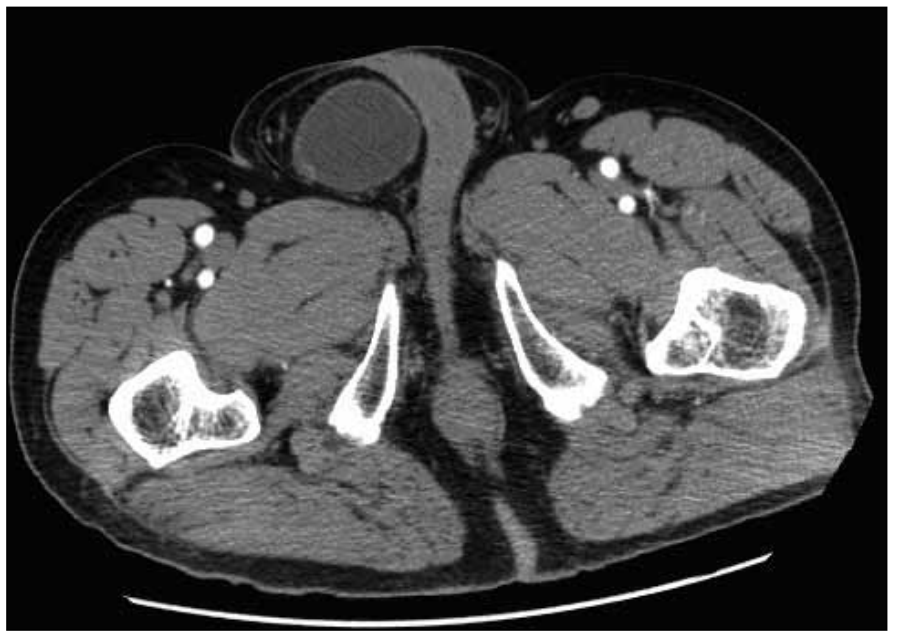

Fig. 1. Right-sided inguinovesical inguinoscrotal bladder hernia.

\section{Case 2}

We report the case of a 54-year-old achondroplastic male who presented to hospital with acute kidney injury. His creatinine was $200 \mu \mathrm{mol} / \mathrm{L}$ on presentation. A renal ultrasound was performed which demonstrated bilateral hydronephrosis and cortical thinning. A CT scan showed severe bilateral hydronephrosis and hydroureter secondary to bilateral inguinal ureteric herniations. In Fig. 3 the herniations with red asterisks indicate the ureters proximally and the yellow asterisks denote the ureters distally.

The patient denied hematuria, frequency, urgency, dysuria, bowel symptoms, fevers, chills and abdominal pain. His medical history was significant for achondroplasia, hearing loss and polycystic kidney disease. Moreover, his family history was significant for Alport syndrome and renal cell carcinoma in his mother.

On examination, the patient's body habitus was consistent with achondroplasia with a BMI of 39 . He had obvious bilateral inguinal hernias in both the supine and standing positions. His abdomen was soft, non-tender and nondistended. There was no organomegaly.

Bilateral retrograde ureteric stent placement was attempted at a peripheral hospital. Due to the tortuosity of the ureters bilaterally, the procedure was unsuccessful. The patient was referred to a tertiary care centre for further evaluation and definitive treatment. Stent placement was attempted a second time and failed again due to the tortuous ureter (Fig. 4).

The patient was referred to interventional radiology for antegrade stent placement. Antegrade stents could not be placed (Fig. 5). Bilateral nephrostomy tubes were then inserted.

The patient's creatinine decreased from $200 \mu \mathrm{mol} / \mathrm{L}$ to his baseline, $120 \mu \mathrm{mol} / \mathrm{L}$. The patient was discharged with

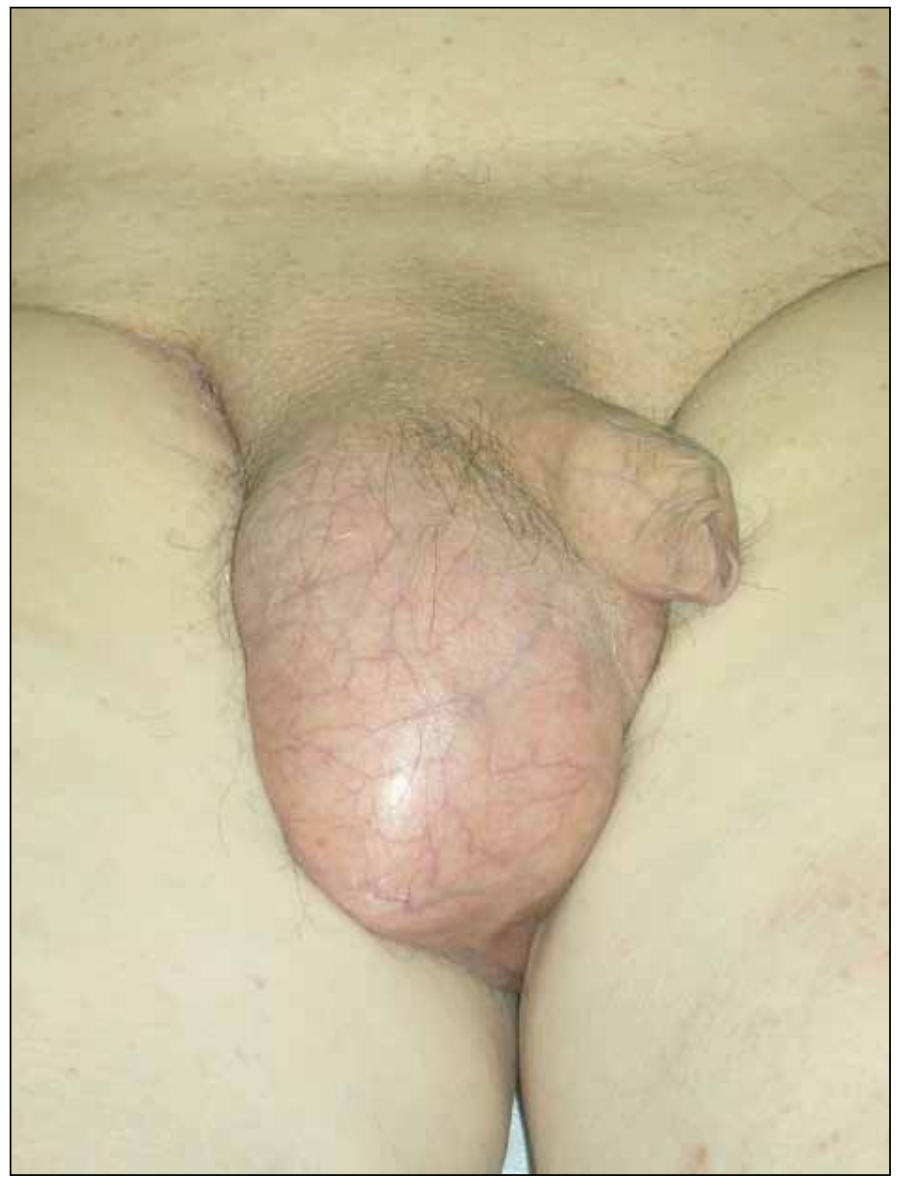

Fig. 2. Preoperative appearance.

nephrostomy tubes. He underwent a bilateral ureteric reimplantation and bilateral inguinal hernia repairs during the same operation with no complications.

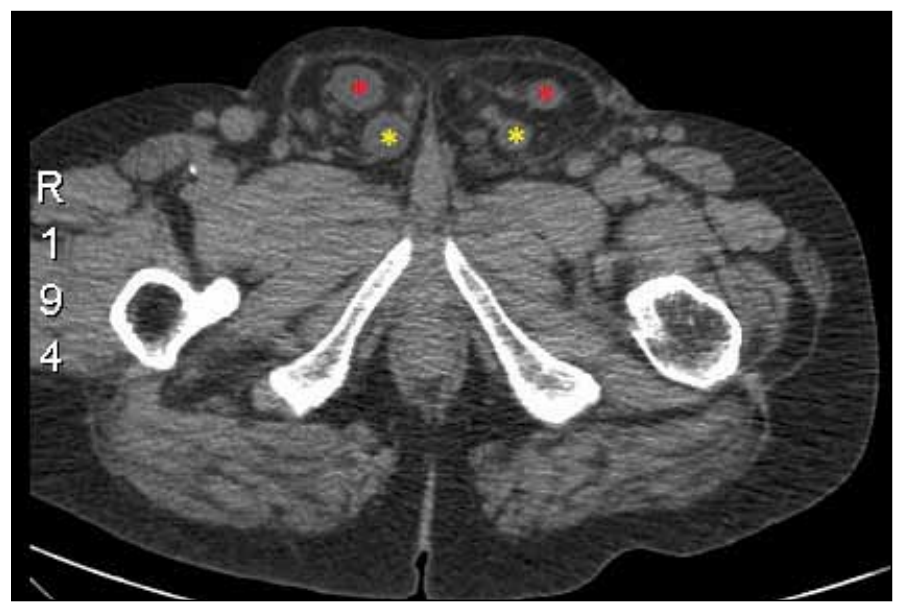

Fig. 3. A computed tomography scan demonstrating bilateral ureteric herniations. 


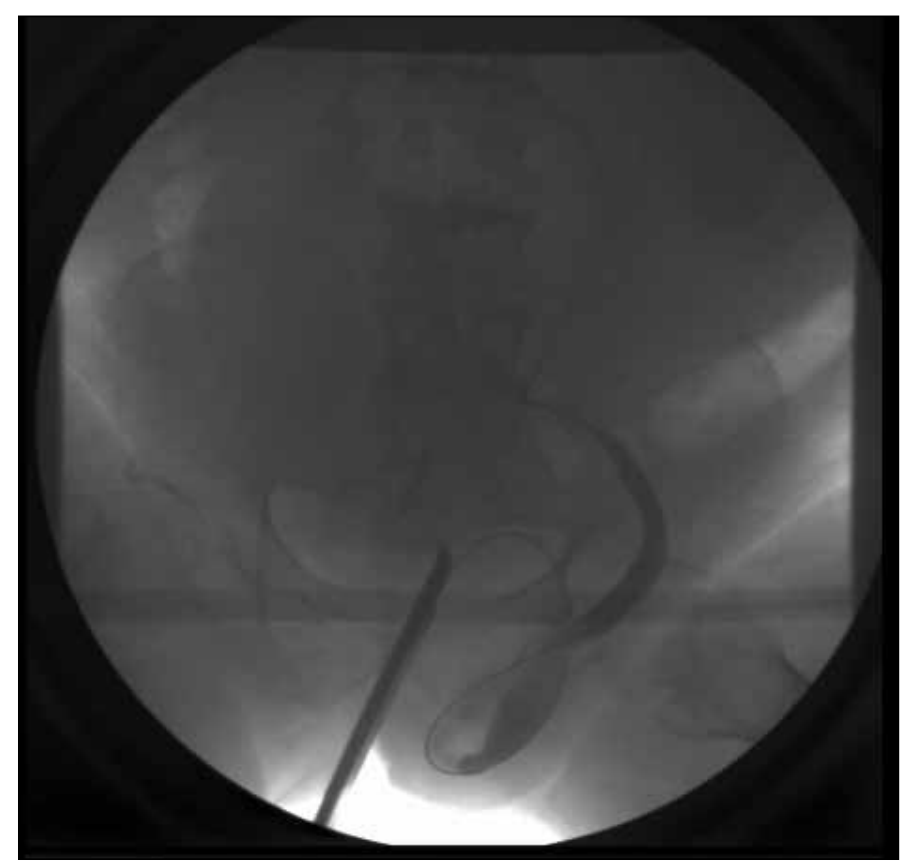

Fig. 4. Attempted left retrograde pyelogram demonstrating the tortuous left ureter.

\section{Discussion}

Inguinoscrotal bladder hernias are rare, with less than 100 reported cases. ${ }^{4}$ However, it is reported that as many as $4 \%$ of inguinal hernias involve the bladder. ${ }^{5}$

Case 1 was an atypical presentation. The patient applied pressure to his scrotum to initiate micturition. Based on his history and physical exam, a CT scan was obtained. A CT scan and ultrasound were reliable to confirm diagnosis. ${ }^{6-9}$ Considering that the patient's pain resolved over a short period, it was decided that elective surgery (performed by urologic and general surgeons) was the best option to repair the hernia. In this case, excising the bladder from the hernia was difficult, consistent with the chronicity of the defect. After a dissection of the bladder and a ureteric re-implantation, the hernia was successfully repaired. The patient recovered with minimal complications.

Case 2 involved bilateral ureteroinguinal herniations. The two types of ureteroinguinal herniations described in the literature are paraperitoneal and extraperitoneal. ${ }^{10}$ Paraperitoneal hernias comprise $80 \%$ of inguinoscrotal ureteric hernias and are thought to arise due to adherence of the ureter to the hernia sac posteriorly. ${ }^{10}$ Extraperitoneal hernias comprise $20 \%$ of ureteroinguinal hernias and contain the ureter without any hernia sac. This type of herniation occurs due to the abnormal development of the ureter from the Wolffian duct or adhesions to the genito-inguinal ligaments, which cause the ureters to descend into the scrotum with the testicles. ${ }^{10}$
When evaluating patients with inguinal hernias, it is important to elicit a detailed history to identify urologic organ involvement. Lower urinary tract symptoms, hematuria, acute urinary obstruction, double phase micturition requiring pressure to initiate or finish voiding, or ipsilateral flank pain with an inguinal hernia may indicate urologic organ involvement and should prompt further investigations with an ultrasound and/or CT scan. If left untreated, inguinal hernias can lead to strangulatation of the bladder or ureter, which would require surgical resection with ureteric reimplantation. Depending on the length of ureter involvement, options for repair would include ureteroneocystostomy, psoas hitch, Boari flap or possibly transureteroureterostomy. Also, the ipsilateral kidney may become obstructed and nonfunctional and serve as a nidus for recurrent pyelonephritis.

If urologic organ involvement is not identified prior to surgical repair, there may be a higher risk of iatrogenic damage to the bladder, ureter or adjacent organs; therefore, identifi-

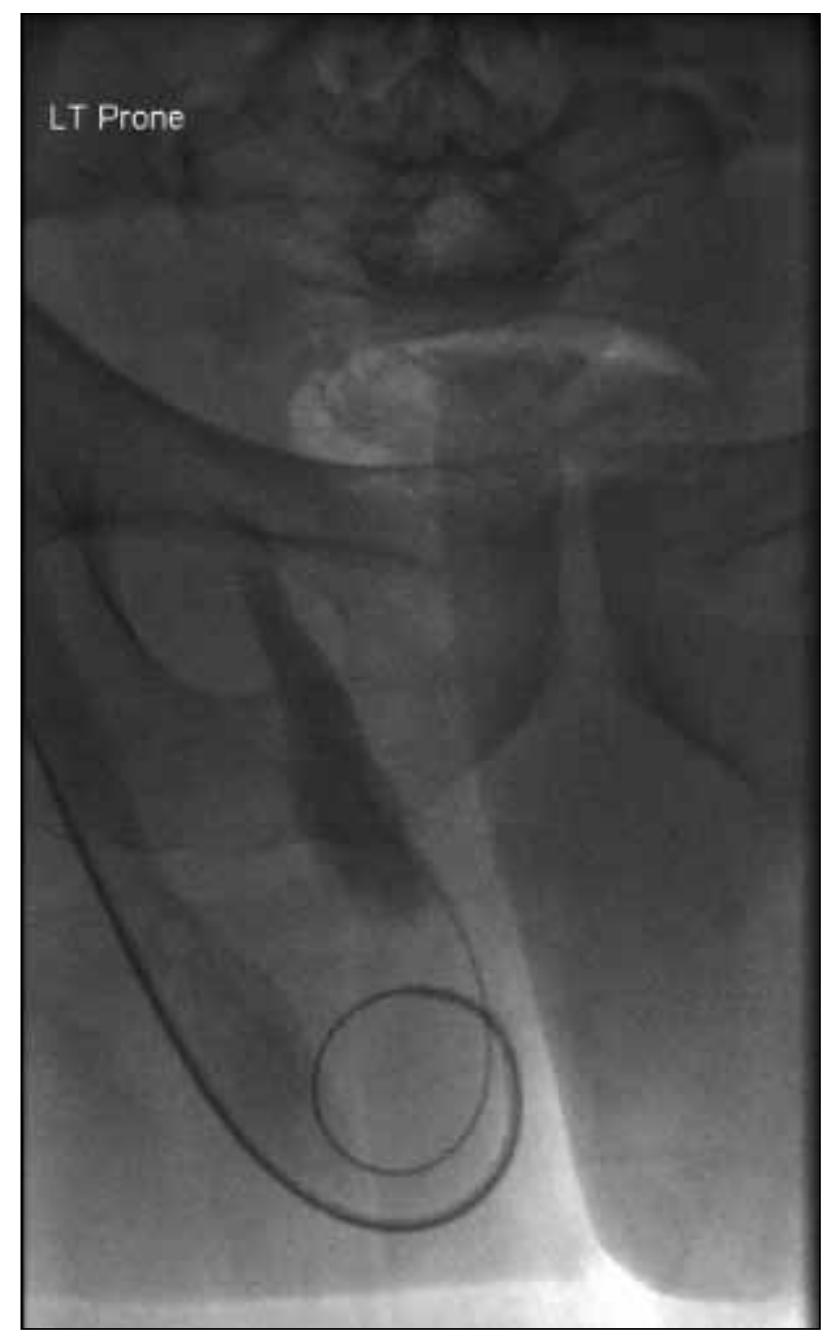

Fig. 5. Attempted antegrade stent placement with patient in the prone position. 
cation of urologic organ involvement in inguinal hernias is crucial to prevent surgical complications and to avoid complications if left untreated.

\section{Conclusion}

Large inguinoscrotal hernias remain rare. A detailed urological history should be performed with any inguinal hernia to rule out bladder or ureteric involvement. When inguinal hernias are suspected on history and physical exam, an ultrasound or CT should be obtained to confirm diagnosis. Surgery should be considered to prevent bladder, ureteric and renal complications and to resolve presenting symptoms. A multidisciplinary approach between Urology and General Surgery departments should also be considered.

Competing interests: Dr. McKay, Dr. Organ, Dr. Bagnell, Dr. Gallant and Dr. French delare no competing financial or personal interests.

This paper has been peer-reviewed.

\section{References}

1. Oruc MT, Akbulut Z, Ozozan 0, et al. Urological findings in inguinal hernias: A case report and review of the literature. Hernia 2004;8:76-9. http://dx.doi.org/10.1007/s10029-003-0157-6

2. Bisharat $M, O^{\prime}$ Donnell $M E$, Thompson $T$, et al. Complications of inguinoscrotal bladder hernias: A case series. Hernia 2009;13:81-4. http://dx.doi.org/10.1007/s10029-008-0389-6

3. Sharma RK, Murari K, Kumar V, et al. Inguinoscrotal extraperitoneal herniation of a ureter. Can I Surg 2009:52:E27-8.

4. Zajaczkowski T. Scrotal Bladder hernia: Report of two cases. Int Urol Nephrol 2007;39:479-84. http:// dx.doi.org/10.1007/s11255-006-9028-2

5. Gomella $L G$, Spires $S M$, Burton JM, et al. The surgical implications of herniation of the urinary bladder. Arch Surg 1985;120:964-7. http://dx.doi.org/10.1001/archsurg.1985.01390320084018

6. Storm DW, Drinis $S$. Radiographic diagnosis of a large inguinal hernia involving the urinary bladder and causing obstructive renal failure. Urology 2008;72:523. http://dx.doi.org/10.1016/j.urology.2008.03.059

7. Rambaransingh B, Docktor B, Ahuja A. Answer to case of the month \#119. Computed tomography findings of a bladder hernia. Can Assoc Radiol J 2007;58:176-7.

8. Casas JD, Mariscal A, Barluenga E. Scrotal cystocele: US and CT findings in two cases. Comput Med Imaging Graph 1998;22:53-6. http://dx.doi.org/10.1016/S0895-6111(98)00007-X

9. Bisharat $M, O^{\prime}$ Donnell $M E$, Thompson $T$, et al. Complications of inguinoscrotal bladder hernias: A case series. Hernia 2009;13:81-4. Epub 2008 Jun 14.

10. Giglio $M$, Medica $M$, Germinale F, et al. Scrotal extraperitoneal hernia of the ureter: Case report and literature review. Urol Int 2001;66:166-8. http://dx.doi.org/10.1159/000056601

Correspondence: Dr. Jeffrey Peter McKay, Department of Urology, Dalhousie University, Halifax, NS; jeff.mckay@dal.co 